\title{
Former les mentors pour entrepreneurs pour aller au-delà de la transmission d'expérience ${ }^{1}$
}

\author{
Étienne St-Jean ${ }^{2}$, Ph.D. \\ Ph.D., professeur \\ Institut de recherche sur les PME \\ Université du Québec à Trois-Rivières \\ 3351, boul. des Forges \\ Trois-Rivières (Québec) \\ Canada \\ G9A 5H7 \\ Tél : 1-819-376-5011 \#4293 \\ etienne.st-jean@uqtr.ca \\ Stéphanie Mitrano-Méda, Ph.D. \\ MERKAPT \\ France \\ smitrano@merkapt.com
}

\footnotetext{
1 St-Jean, É. \& Mitrano-Méda, S. (2013). « Former les mentors pour entrepreneurs pour aller au-delà de la transmission d'expérience ", Revue de l'Entrepreneuriat, Vol. 12, pp. 121-140. https://doi.org/10.3917/entre.121.0121

2 Auteur pour la correspondance
} 


\title{
Résumé
}

Le mentorat entrepreneurial est une pratique d'accompagnement des entrepreneurs novices par des gens d'affaires expérimentés qui gagne en popularité auprès des organismes de soutien à l'entrepreneuriat. Toutefois, une question demeure : est-il nécessaire pour les structures d'accompagnement de former leurs mentors afin de maximiser les apprentissages du novice ou bien l'expérience du mentor devrait suffire ? Pour répondre à ce questionnement, nous avons réalisé une analyse en dyade de l'effet des formations, de l'expérience en entrepreneuriat et en mentorat du mentor sur le style d'intervention et les fonctions du mentor, ainsi que la satisfaction et l'apprentissage du novice. Nos résultats montrent que plus le mentor est formé, plus il développe des compétences relationnelles et, conséquemment, plus il crée un environnement favorable (confiance) et un style d'accompagnement adapté (maïeutique) permettant l'apprentissage et l'autonomisation de l'entrepreneur mentoré. L'expérience du monde des affaires du mentor n'a pas d'impact sur la qualité de la relation ou sur l'apprentissage du novice. Nos résultats montrent que contrairement à nos attentes, l'expérience en mentorat a un impact négatif sur la plupart des fonctions psychologiques du mentor, lequel effet peut être neutralisé par de la formation continue, suggérant aux structures d'accompagnement des formations ciblées auprès de cette clientèle.

Mots clés: mentorat, formation, entrepreneurs, accompagnement, gestion des programmes de soutien.

\begin{abstract}
Entrepreneurial mentoring is the support of novice entrepreneurs by experienced businesspeople professionals that quickly gain popularity with governmental and nongovernmental organizations supporting and developing entrepreneurship. However, a question remains: is it necessary for these organizations to train mentors in order to maximize the novice's learning or is the mentor's experience sufficient? To answer this question, we analyzed (via mentor-mentee dyads) the effect of the mentor's training, entrepreneurship and mentoring experience on his style of intervention and mentor functions, as well as the effect on the mentee's degree of satisfaction and learning. Our results show that the more a mentor is trained, the more he develops relational competencies, thereby creating a favorable (trusting) environment and developing an appropriate style (maieutic), which allows the mentee to learn and become more autonomous in his entrepreneurship practice. However, the mentor's experience in entrepreneurship does not have an impact on the quality of the mentoring relationship nor does it impact the novice's learning. Our results also show that, contrary to our expectations, mentoring experience has a negative impact on most of the psychological functions of the mentor. We found that this effect can be neutralized by the continuous training of mentors, which suggests that entrepreneurship support organizations should implement specific training sessions for senior mentors.
\end{abstract}

Keywords: mentoring, training, entrepreneurs, entrepreneur's support programs management; 


\section{Introduction}

Depuis quelques années, de nombreuses formules d'accompagnement ont vu le jour dans la plupart des pays industrialisés, avec pour objectif de soutenir les entrepreneurs dans la période de démarrage ou pour faciliter la croissance subséquente de leur entreprise. Parmi l'éventail des formules proposées par les pouvoirs publics et les organismes d'aide à l'entrepreneuriat, le mentorat gagne en popularité. Essentiellement, une relation de mentorat consiste à jumeler un entrepreneur expérimenté avec un novice, c'est-à-dire un entrepreneur sans expérience préalable. Les recherches dans ce domaine indiquent que le mentorat permet à l'entrepreneur novice de développer des apprentissages cognitifs et affectifs, parmi ceux-ci la capacité à reconnaître des opportunités et à développer une vision cohérente de leur projet d'affaire (Ozgen et Baron, 2007; St-Jean et Audet, 2012; Ucbasaran et al., 2009).

Du point de vue des organismes qui coordonnent de tels programmes, l'intérêt se porte non seulement sur les retombées pour le novice, mais également sur la « boîte noire » du mentorat. En effet, si globalement le mentorat semble donner des résultats positifs (p. ex. Cull, 2006; Deakins et al., 1998; Gravells, 2006; Nandram, 2003; Wikholm et al., 2005), il est démontré que certaines relations de mentorat, dans d'autres contextes que l'entrepreneuriat, peuvent déboucher sur des comportements inadéquats de la part du mentor, menant parfois à des conséquences peu souhaitables pour le mentoré (Eby et al., 2004; Simon et Eby, 2003). Il est alors possible d'envisager que des mentors d'entrepreneurs novices pourraient être inadéquats, voire nuisibles, dans les fonctions que l'on pourrait s'attendre d'eux. Au-delà des comportements nuisibles, certains mentors pourraient n'être que peu utiles, offrant alors un accompagnement produisant un effet marginal (p. ex. Ragins et al., 2000).

Dans cette perspective, on peut se demander si de devenir un mentor efficace implique une préparation adéquate et, du coup, nécessite une formation préalable, ou si le simple fait d'avoir une expérience personnelle ou professionnelle en entrepreneuriat est suffisante pour que cela produise des résultats positifs pour le mentoré. À cet effet, il semble qu'aucune étude n'ait démontré la conséquence de recruter certains profils de mentor et de les former sur les résultats évoqués par le mentoré. C'est sur cette problématique que cette communication s'attardera. Pour y parvenir, nous allons tout d'abord faire un état des lieux de la littérature sur le mentorat et sur le rôle des profils de mentors et de la formation de ceux-ci dans ce cadre, puis nous aborderons et discuterons des résultats de l'étude empirique menée sur cette problématique. Une discussion ainsi que des recommandations pour les organismes viendront conclure cette communication.

\section{Examen de la documentation}

Dans cette section, nous présenterons d'abord une définition du mentorat pour entrepreneur. Par la suite, il sera question des principaux éléments du cadre conceptuel du mentorat pour entrepreneur, avant d'aborder spécifiquement le rôle de la formation des mentors et de terminer avec le rôle de leurs profils.

\subsection{Définition du mentorat}


Dans notre monde contemporain et en s'inspirant de la métaphore grecque ${ }^{3}$, un mentor est généralement une personne possédant certaines qualités ou qui est en position d'autorité et qui veille de façon bienveillante sur un individu plus jeune, ou moins expérimenté, lequel bénéficie des conseils et du soutien de son mentor. Dans le contexte de l'entrepreneuriat, bien qu'il puisse exister plusieurs définitions, le mentorat concerne l'établissement d'une relation de soutien entre un entrepreneur novice (nommé le mentoré) et une personne expérimenté du monde des affaires (appelé le mentor), le dernier permettant au premier de se développer en tant que personne. Tel que le suggère Paul (2004), le mentorat se distinguerait du coaching, du tutorat ou du compagnonnage du fait qu'il est davantage orienté vers la quête de sens plutôt que l'acquisition de techniques. Tout en étant ancré davantage vers l'action, contrairement au counselling, au conseil ou à la médiation, il se distinguerait quand même du parrainage, en étant légèrement moins orienté vers l'action que cette forme d'accompagnement. Ainsi, l'idée sous-jacente au mentorat serait l'éducation, où le mentor est dans un registre de « conduite » et de « guidage », et se distingue du coaching, basé sur l'idée d'entraînement, ou du counselling, axé sur une relation d'aide psychologique (Deschamps et al., 2010). Pour reconnaître une relation de mentorat, tel que le proposent Haggard et al. (2011), trois dimensions doivent être présentes : il doit y avoir une relation de réciprocité, possédant des bénéfices développementaux pour l'accompagné, en particulier pour la carrière de celui-ci, ainsi que des interactions régulières et substantielles dans une perspective à long terme. Il convient de rappeler que ces différentes postures théoriques peuvent évoluer dans la pratique et amener un accompagnateur à changer de posture en cours de relation, ajoutant une confusion supplémentaire entre les rôles et objectifs attendus d'une relation de mentorat (Paul, 2004).

\subsection{Cadre conceptuel du mentorat}

Dans une recension exhaustive de la littérature sur le mentorat en contexte organisationnel, Wanberg, Welsh, \& Hezlett (2003) font état de différentes dimensions permettant de développer des retombées chez le protégé ${ }^{4}$. D'abord, autant le protégé que son mentor contribuent à la réussite de la relation de mentorat, laquelle possède certaines caractéristiques qui peuvent aussi s'avérer déterminantes. La dynamique qui s’installe permettra au mentor d'exercer ses fonctions, ce qui permettra au mentoré de générer différents apprentissages, lesquels feront en sorte de développer des retombées distales telles la satisfaction à l'emploi, la rétention à la profession, les progressions salariales et dans la hiérarchie, et ainsi de suite.

Dans différents contextes de mentorat, il est largement démontré que la confiance est essentielle pour permettre à la relation de se développer et, du coup, de maximiser le potentiel de développement pour le mentoré (Kram, 1985; Ragins, 1997). Aussi, les membres de la dyade doivent avoir une "chimie » positive entre eux (Couteret et al., 2006; Cull, 2006). Cela pourrait faire référence à la similitude perçue à l'égard du mentor,

\footnotetext{
3 Basée sur L'Odyssée, un récit du dramaturge grec Homère, dans lequel il raconte le rôle de Mentor dans l'éducation de Télémaque, le fils d'Ulysse, en l'absence de ce dernier.

${ }^{4}$ Le terme protégé est utilisé dans la littérature pour désigner celui qu'accompagne un mentor dans un contexte organisationnel, alors que le mentoré désigne spécifiquement l'entrepreneur accompagné par un mentor.
} 
une composante essentielle pour assurer le succès de la relation (Allen et Eby, 2003; Ensher et Murphy, 1997; Wanberg et al., 2006). Ces éléments sont des conditions essentielles mais non suffisantes pour que des retombées surviennent chez le mentoré.

Les fonctions du mentor font référence, d'une certaine manière, aux différents rôles joués par le mentor dans la relation de mentorat (Kram, 1985). Une fois la confiance établie, c'est grâce aux fonctions du mentor que le mentoré développera des retombées de sa relation (Wanberg et al., 2003), dont différents apprentissages, souvent considérés comme les plus importantes retombées (Allen et Eby, 2003). Les différentes fonctions du mentor étudiées dans la grande organisation se regroupent généralement en trois grandes catégories : les fonctions psychologiques, celles relatives à la carrière et la fonction de modèle de rôle (Pellegrini et Scandura, 2005; Scandura et Williams, 2001). Dans le contexte de l'entrepreneur novice, le contenu des fonctions est différent de celui des mentors en grande organisation mais elles se regroupent toutefois selon ces mêmes catégories (St-Jean, 2010). Ainsi, il y a les fonctions psychologiques, c'est-à-dire lorsque le mentor donne de la rétroaction à son mentoré, lorsqu'il le sécurise, lorsqu'il cherche à le remotiver et lorsqu'il sert de confident. Il y a aussi les fonctions reliées à la carrière d'entrepreneur lorsque le mentor transmet de l'information sur le monde des affaires, lorsqu'il présente le mentoré à des personnes clés du milieu, lorsqu'il confronte les idées du mentoré afin de s'assurer que celles-ci sont bien mûries et lorsqu'il le guide vers des solutions appropriées. Finalement, il y a la fonction de modèle lorsque le mentor confie au mentoré ses expériences du monde des affaires afin de lui donner des exemples inspirants. Là aussi, il semble utile que les mentors comprennent les différentes fonctions qu'ils sont appelés à exercer auprès des novices afin qu'ils puissent être plus efficaces dans leur intervention.

Pour maximiser les différentes retombées, il est reconnu que dans un contexte de mentorat entrepreneurial, le mentor devrait idéalement exhiber un style maïeutique (c.-à-d. axé sur le questionnement du mentoré), et cela couplé d'un engagement important dans sa relation de mentorat (St-Jean et Audet, 2011). Dans un tel contexte, cela fait du sens puisque le mentoré doit conserver son autonomie décisionnelle et apprendre à trouver des solutions par lui-même. En outre, les mentors étant des généralistes (contrairement aux coachs, qui sont des spécialistes, par exemple), il est préférable qu'ils demeurent dans une posture axée sur le questionnement, évitant alors de fournir de mauvais conseils au mentoré, ne connaissant pas les tenants et aboutissants de chaque secteur industriel. Or, il appert qu'un nombre important de mentors sont plutôt directifs (à l'opposé de maïeutique) et peu engagés dans la relation (St-Jean et Audet, 2011). Sachant que cela nuit considérablement au développement des retombées de la relation, notamment au niveau du développement de l'auto-efficacité entrepreneuriale et des différents apprentissages du mentoré, il est donc souhaitable que les mentors apprennent à développer un style qui réponde davantage aux besoins des mentorés.

Finalement, il est démontré que le contact d'un entrepreneur novice avec un mentor lui permet d'augmenter ses apprentissages (Florén, 2003; St-Jean et Audet, 2012). Selon Choueke et Armstrong (1998), le mentorat constitue la quatrième source d'apprentissage en importance (43\%), devançant même les études supérieures. Selon Clutterbuck et Megginson (1999), le mentor apporte au mentoré un espace de réflexivité personnelle dans laquelle ce dernier prend l'opportunité de développer des nouvelles idées au travers 
d'activités de réflexion ininterrompues et déterminées. Ainsi, cela lui permet de faire du sens des événements critiques qu'il vit dans son entreprise (Cope et Watts, 2000), ce qui met en relief l'importance du mentorat pour l'entrepreneur novice.

\subsection{Rôle de la formation des mentors}

La plupart des chercheurs sur le mentorat intra-organisationnel s'accordent sur le besoin de former les participants d'un programme de mentorat formel afin de les préparer à la relation d'accompagnement (Allen et al., 2009; Baugh et Fagenson-Eland, 2007; Clutterbuck et Megginson, 1999; Cranwell-Ward et al., 2004; Hattingh et al., 2007; Wilson et Elman, 1990). Les participants, aussi bien les mentors que les mentorés, doivent être préparés à leurs rôles et doivent comprendre le cadre défini par l'organisation coordinatrice du programme. Les recherches ont montré que les relations ont trois fois plus de chance de réussir si le mentoré et le mentor ont été formés (Cranwell-Ward et al., 2004: p. 99). Et selon Allen et al. (2009: p. 60), la formation des mentors et des mentorés a été associée à un plus fort engagement des mentors, une meilleure compréhension du programme et de la perception de ses résultats.

Les formations de mentors agissent à plusieurs niveaux : elles définissent le cadre et les règles du jeu de la relation, décrivant bien les rôles et responsabilités de chacun, et elles aident les mentors à développer les compétences relationnelles nécessaires pour un meilleur transfert d'expérience, par exemple dans un style maïeutique pour les mentors d'entrepreneurs.

Définir le cadre est essentiel au démarrage de la relation car cela peut éviter certains dysfonctionnements évoqués par Eby (2007) et certaines des frustrations évoquées par les mentorés (Baugh et Fagenson-Eland, 2007) lorsque le mentor sort du cadre et devient trop dirigiste. Le cadre permet au mentor, d'une part, de bien comprendre son rôle, sa relation avec le mentoré, ses fonctions, et de faire la différence entre mentorat, coaching, formation, conseil et autres formes d'accompagnement, et d'autre part, de connaître les limites de son intervention. La formation permet également aux mentors de développer des compétences relationnelles d'accompagnement nécessaires à l'établissement d'une relation de qualité. Ces compétences pourraient ne pas avoir été acquises dans leur parcours professionnel passé, telles que la communication, le questionnement, l'écoute et la création d'une relation de confiance (Cranwell-Ward et al., 2004).

Lorsque l'on considère plus spécifiquement le contexte de mentorat entrepreneurial et les fonctions particulières du mentor (St-Jean, 2010), il apparaît évident que son expérience du monde des affaires, et en particulier d'entrepreneur, lui permet de remplir les fonctions dites de carrière : intégration du mentoré dans les réseaux, transfert d'informations sur les affaires ou d'un secteur d'activité, confrontation des idées et guide pour le développement de l'entreprise. La fonction de modèle de rôle, en tant qu'entrepreneur expérimenté, n'aurait pas non plus besoin de préparation préalable pour qu'elle s'exerce auprès des novices. Cependant, en ce qui concerne les fonctions dites psychologiques, l'expérience n'est pas forcément un indicateur de capacité à les fournir. Les fonctions psychologiques (servir de réflecteur, sécurisation du mentoré, motivation, et confident) sont directement liées aux compétences relationnelles évoquées plus haut. 
Somme toute, la littérature sur le mentorat intra-organisationnel nous suggère que les mentors doivent être formés pour comprendre le cadre de la relation et développer des compétences relationnelles, afin d'avoir des relations de mentorat efficaces. De plus, une partie importante des fonctions du mentorat entrepreneurial nécessite des compétences relationnelles qui n'ont pas été nécessairement acquises dans le parcours professionnel du mentor. Et finalement, il est important que le mentor soit préparé à accompagner l'apprentissage sur le tas (ou expérientiel) du mentoré. Ces considérations amènent l'hypothèse suivante

H1 : La formation du mentor a un effet positif sur la relation développée avec le mentoré ainsi que sur le style d'intervention, les fonctions psychologiques et l'apprentissage du novice.

\subsection{Le rôle du profil du mentor}

Dans la forte majorité des programmes de mentorat mis en place par les structures d'accompagnement de plusieurs pays, les mentors sont des bénévoles issus du monde des affaires. Pour les organismes de soutien à l'entrepreneuriat, une telle réalité possède des avantages évidents, notamment d'abaisser les coûts de l'accompagnement, mais aussi des inconvénients majeurs. En effet, il est plus difficile d'encadrer un bénévole qui veut aider que d'imposer la marche à suivre à un salarié que l'on recrute sur la base d'un profil recherché. Dans ce contexte, pour s'assurer d'avoir suffisamment de mentors bénévoles pour offrir le service d'accompagnement, les organismes acceptent non seulement des mentors ayant de l'expérience en affaires comme entrepreneur, mais également d'autres qui n'ont jamais démarré d'entreprises, tels des banquiers, des conseillers en développement d'entreprises, des coachs professionnels, des hauts dirigeants de grandes corporations, etc.

Or, on pourrait se demander si l'expérience du monde des affaires du mentor en tant qu'entrepreneur pourrait influencer les différentes composantes de la relation de mentorat. Par exemple, sachant que la fonction de modèle de rôle est plus marquée chez les mentors ayant été eux-mêmes entrepreneurs en comparaison avec les autres (St-Jean, 2010), il est possible que cela ait un effet sur l'apprentissage de l'entrepreneur mentoré. Un autre exemple, sachant que les mentors sont susceptibles d'aider les mentorés à identifier des opportunités (Ozgen et Baron, 2007; Ucbasaran et al., 2009), il serait logique de penser qu'un mentor ayant de l'expérience à identifier lui-même des opportunités serait à même d'aider davantage le novice à développer des schémas cognitifs à cet égard (Baron et Ensley, 2006). Par contre, tel que le suggèrent St-Jean et Audet (2011), il pourrait être plus difficile pour un entrepreneur expérimenté d'adopter un style maïeutique (i.e. axé sur les questionnements) puisque leur expérience leur permet d'identifier rapidement des « réponses » à transmettre au novice, donc d'user d'un style plus directif. Malgré cela, sachant que la similitude est importante dans l'établissement de la relation (Lankau et al., 2005) et qu'un mentor n'ayant jamais été entrepreneur pourrait être considéré moins pertinent aux yeux du mentoré, nous proposons les hypothèses suivantes : 
H2 : L'expérience du monde des affaires du mentor en tant qu'entrepreneur a un effet positif sur la relation développée avec le mentoré ainsi que sur les fonctions et l'apprentissage du novice.

H3 : L'expérience du monde des affaires du mentor en tant qu'entrepreneur a un effet négatif sur le style d'intervention du mentor.

De plus, l'expérience de mentorat que va accumuler le mentor pourrait aussi lui être bénéfique. En effet, intuitivement, il est tout à fait logique de prétendre qu'un mentor qui en serait à sa première expérience pourrait être moins efficace qu'un autre qui serait habitué à accompagner des entrepreneurs. D'ailleurs, plusieurs recherches prennent en considération l'expérience de mentorat comme une variable exogène dont il faut contrôler les effets sur les retombées du mentorat (p. ex. Allen et Eby, 2003; Bauer, 1999; Ragins et al., 2000). De manière particulière, dans un contexte de grande organisation, il est démontré que l'expérience passée de mentorat permet au mentor d'exercer avec davantage d'intensité ses fonctions de carrière (Fagenson-Eland et al., 1997) et parfois également des fonctions psychologiques (Allen et Eby, 2004). Sachant que l'intensité des fonctions du mentor est reliée aux retombées, notamment les apprentissages, tout porte à croire que l'expérience de mentorat pourrait procurer de plus grandes retombées au mentoré. Dans la même logique, un mentor expérimenté dans l'accompagnement pourrait apprendre de ses erreurs passées et améliorer sa capacité à mettre en place une relation de confiance, tout en adoptant davantage un style d'intervention qui est plus adapté aux entrepreneurs (i.e. maïeutique et engagé). Ces considérations amènent l'hypothèse suivante :

H4 : L'expérience passée de mentorat a un effet positif sur la relation développée avec le mentoré ainsi que sur le style d'intervention, les fonctions et l'apprentissage du novice.

\section{Méthodologie}

Dans cette section, nous aborderons d'abord le programme qui a été étudié afin de tester nos hypothèses. Par la suite, il sera question de l'échantillonnage, avant de compléter avec les mesures utilisées.

\subsection{Le programme de mentorat pour entrepreneur étudié}

Le Réseau M, qui sert de toile de fond à la présente recherche, a été créé en 2000 par la Fondation de l'entrepreneurship, un organisme voué au développement économique sur le territoire du Québec. Il est offert aux entrepreneurs novices par le biais d'environs 70 cellules de mentorat dispersées sur l'ensemble du territoire du Québec. Ces cellules sont généralement soutenues par des organismes de développement économique tels les Centres locaux de développement (CLD), les Sociétés d'aide au développement des collectivités (SADC) et les chambres de commerce locales. Celles-ci assurent le développement du programme au niveau local ou régional, tout en adhérant au modèle du mentorat d'affaires développé par la Fondation. De façon plus précise, les organismes locaux se dotent d'un coordonnateur de la cellule, lequel est responsable du recrutement des mentors bénévoles, de l'organisation des séances de formation à leur intention, de la promotion du programme auprès des entrepreneurs novices, du jumelage des participants 
et de la supervision de la relation qui s'ensuit. Les entrepreneurs novices peuvent bénéficier du soutien d'un mentor pour un coût minime, soit quelques centaines de dollars annuellement, et parfois même gratuitement. Basé sur un code d'éthique d'intervention où la confidentialité des relations est d'une importance capitale, le Réseau M s'est également doté d'un contrat-type pour encadrer et guider les parties dans le choix des modalités de leur relation et des objectifs à atteindre. Il s'agit donc d'un mentorat de type « formel ».

Afin de bien encadrer le développement local, la Fondation de l'entrepreneurship développe des ateliers de perfectionnement orientés sur la relation mentor-mentoré pour éclairer concrètement le rôle des mentors auprès des nouveaux entrepreneurs. Ainsi, différentes formations offertes par le Réseau M permettent aux mentors de clarifier le style d'intervention idéal qu'il doit privilégier, ainsi que ses rôles, sans compter des activités qui sont organisées pour leur permettre de développer leur capacité d'écoute et de questionnement, et ainsi de suite. Certaines cellules suggèrent à leurs mentors de suivre une formation de base ( 3 heures) sur les rôles du mentor avant d'être jumelés alors que d'autres ne l'exigent pas.

\section{2 Échantillonnage et construction de l'analyse en dyade}

Au printemps 2008, les 981 mentorés du Réseau M de la Fondation de l'entrepreneurship possédant une adresse de courriel valide ont été sollicités par ce moyen pour participer à une recherche. De ce nombre, 362 ont répondu au questionnaire en ligne, pour un taux de réponse de 36,9\%. Ils devaient répondre à de nombreuses questions, incluant des caractéristiques sociodémographiques et concernant leur entreprise, leur profil psychosociologique, leur relation de mentorat, leur perception à l'égard de leur mentor et certaines retombées du mentorat. Nous leur avons également demandé s'ils acceptaient que les réponses de leur mentor puissent être jumelées de manière anonyme aux leurs, permettant ainsi des analyses en dyade. Sur les 362 répondants, 216 mentorés ont accepté que l'on procède au jumelage et indiqué le nom de leur mentor.

Au printemps 2010, le Réseau M de la Fondation de l'entrepreneurship a fourni une liste de 1004 mentors possédant une adresse de courriel valide. À noter que selon les estimations du Réseau M, il y aurait entre 1200 et 1300 mentors. Chaque mentor possédant une adresse courriel a reçu une invitation personnalisée à répondre à un questionnaire en ligne ainsi que deux rappels pour les non-répondants. Au total, 366 mentors ont accepté l'invitation, pour un taux de réponse de $36,4 \%$.

Pour les deux échantillons, nous avons comparé les répondants hâtifs, soit ceux ayant répondu avant le premier rappel à ceux ayant répondu suite à un rappel, suivant l'argument développé par Armstrong et Overton (1977) pour estimer le biais du nonrépondant. Autant pour l'échantillon des mentorés que celui des mentors, en comparant les répondants hâtifs à ceux étant en retard, aucune différence significative n'est apparue pour les deux groupes concernant les caractéristiques sociodémographiques (âge, sexe, scolarité, etc.) ou les variables d'intérêt pour cette recherche. Cela suggère que les nonrépondants pourraient être similaires aux répondants à l'enquête. 
Sur l'ensemble des mentors ayant été identifiés dans l'enquête auprès des mentorés et dont le mentor a répondu à l'enquête de 2010, 78 jumelages en dyade ont été effectués, correspondant à $21,5 \%$ de l'échantillon de mentorés. Ainsi, dans cette recherche en dyade, le mentor et le mentoré répondaient chacun à un questionnaire de manière anonyme et à des moments différents, ce qui exclut toute interférence entre les réponses de l'un vers l'autre.

\subsection{Mesures pour les mentors}

La formation des mentors. L'une des questions posées aux mentors était le nombre d'heures de formation reçues par le biais du Réseau $M$ et à l'extérieur de celui-ci : «Combien d'heures de formation pertinente à votre fonction de mentor avez-vous reçues jusqu'à maintenant (si aucune, veuillez indiquer « $0 »)$ ? ». Ces deux variables sont celles retenues afin de vérifier leur influence sur les composantes de la relation chez les mentorés.

L'expérience en affaires des mentors. Pour connaitre le niveau d'expérience de l'entrepreneuriat des mentors, ils devaient indiquer le nombre d'années où leur principale occupation était « entrepreneur (à son compte, en affaires)».

L'expérience de mentorat. Les mentors ont indiqué le nombre de relations de mentorat de plus de trois (3) rencontres qu'ils ont eu jusqu'à maintenant dans leur implication de mentor au sein du Réseau M.

\subsection{Mesures pour les mentorés}

Au niveau des mentorés, plusieurs mesures ont été utilisées (voir les questions à l'annexe 1) :

Comme indicateur de la qualité de la relation : La confiance envers le mentor (basé sur Rempel et Holmes (1986)) (3 items), la similitude perçue (inspiré de Ensher et al. (2002)) (4 items) et la satisfaction envers le mentor (Ragins et Cotton, 1999) (4 items). Les mesures sont unidimensionnelles et les alphas de Cronbach sont respectivement de 0,741, de 0,897 et de 0,937 .

Comme indicateur du style d'intervention: l'approche maïeutique (3 items) et l'engagement dans la relation (3 items) (deux mesures basées sur St-Jean et Audet (2011)). Les mesures sont unidimensionnelles et les alphas de Cronbach sont de 0,688 et de 0,90 , respectivement.

Comme indicateur des fonctions du mentor : les neuf fonctions du mentor (St-Jean, 2010) (35 items au total). Les mesures sont unidimensionnelles et les alphas de Cronbach varient entre 0,882 et 0,953 .

Comme indicateur de retombée pour le mentoré : l'apprentissage réalisé dans la relation (Allen et Eby, 2003) (5 items). La mesure est unidimensionnelle et l'alpha de Cronbach est de 0,910 . 


\section{Résultats}

Avant d'aborder les résultats, nous présenterons les données descriptives des principales mesures. Ensuite, nous présenterons les analyses qui ont permis de tester les hypothèses posées précédemment. Par la suite, les résultats ayant suscité des interrogations supplémentaires, dans une démarche exploratoire, nous avons procédé à des analyses complémentaires.

\subsection{Portrait de la formation et du profil des mentors}

D'abord, soulignons que le nombre d'heures de formation déclarées dans le Réseau M varie de 0 à plus de 50 heures $^{5}$, tout comme c'est le cas pour les formations à l'extérieur du Réseau M. Certains mentors exceptionnels dépassent les 50 heures de formation, que ce soit dans le Réseau M (7 cas) ou ailleurs (19 cas). Le nombre d'heures moyen de formations suivies dans le Réseau $M$ est de 9,2 (médiane de 8), avec un écart-type de 10,36. Le nombre d'heures moyen de formations suivies à l'extérieur du Réseau M est de 11,56 (médiane de 3), avec un écart-type de 15,54. On peut voir que la distribution ne suit pas une courbe normale, en particulier pour les formations suivies à l'extérieur du Réseau $\mathrm{M}$, où un groupe " extrême » a suivi un très grand nombre d'heures de formation et une grande majorité n'a suivi qu'un petit nombre d'heures, voire pas du tout.

Au niveau de l'expérience en affaires, le nombre d'années des mentors varie de 1 à 54 ans, avec une moyenne de 23,56 ans (médiane 23 ans) et un écart-type de 11,49. La distribution suit une courbe normale.

Pour le nombre de relations de mentorat (i.e. expérience de mentorat), cela varie de $0^{6}$ à 21 , avec une moyenne de 5,96 (médiane 4) et un écart-type de 4,39. La distribution est de type «count», soit une assez grande concentration ayant eu peu de relations et cela déclinant progressivement jusqu'à 21 . En effet, $90 \%$ de l'échantillon a eu moins de 10 relations au total.

\subsection{Effet de la formation, de la carrière du mentor et de son expérience de mentorat chez le mentoré}

Nous avons vérifié d'abord si la formation reçue par les mentors avait une influence sur les réponses du mentoré à l'égard des retombées de la relation, des fonctions et du style que le mentor va déployer ou de la qualité de la relation avec le mentor (hypothèse 1). Les résultats sont très marqués et révélateurs. Comme on peut le constater au Tableau 1 , la formation reçue par le Réseau $M$ et celle prise ailleurs est corrélée positivement et significativement avec deux des trois composantes de la qualité de la relation, soit la

\footnotetext{
5 Il faut mentionner qu'il s'agissait d'une échelle graduée par catégories, où le maximum est « + de 50 heures ».

6 Précisons ici qu'il s'agit des données descriptives de l'échantillon total des mentors, et non celui «en dyade ", afin de fournir le portrait de la situation au Réseau M. Voilà pourquoi nous constatons que certains mentors n'ont jamais eu de relations de mentorat, ce qui ne serait pas le cas chez ceux qui ont été jumelés en dyade puisqu'ils ont nécessairement eu au moins une relation, soit celle pour laquelle l'analyse a été effectuée.
} 
confiance à l'égard du mentor et de la similitude perçue. Toutefois, la formation n'a pas d'effet sur la satisfaction générale à l'égard du mentor. Au niveau du style du mentor, rappelons que le style qui combine une approche maïeutique et un engagement marqué du mentor donne les meilleurs résultats (St-Jean et Audet, 2011).

On peut constater que le fait de suivre des formations via le Réseau M permet au mentor de comprendre le style idéal à adopter et il semble le mettre en pratique puisque les corrélations sont positives et significatives. Les formations hors réseau développent également l'approche maïeutique mais pas l'engagement du mentor. Au niveau des fonctions psychologiques du mentor, elles sont toutes exercées de manière plus marquées lorsque le mentor est davantage formé dans le Réseau M, alors que ce n'est pas le cas pour les formations hors réseau. Pour les autres fonctions, seules celles de guide (c.-à-d. de suggérer des pistes au mentoré) et de modèle de rôle sont plus marquées en formant les mentors dans le Réseau M, alors que ça n'a pas d'effet pour les formations hors réseau. Finalement, on constate que la formation des mentors dans le Réseau M est corrélée positivement et significativement (à $\mathrm{p} \leq 0,10)$ avec l'apprentissage du mentoré, tel que ce dernier va le déclarer. Ces résultats confirment $\mathrm{H} 1$.

$\mathrm{Au}$ niveau de l'expérience en entrepreneuriat du mentor, il n'y a aucune corrélation significative avec les variables d'intérêt. Ainsi, l'expérience du monde des affaires du mentor n'influence pas positivement la qualité de la relation, les fonctions psychologiques, de carrière ni même de modèle de rôle, et est sans effet sur l'apprentissage. Nous ne pouvons donc pas accepter l'hypothèse 2 (H2). Également, il n'y a aucune relation entre le style d'intervention du mentor et sa carrière (via le nombre d'années d'expérience en affaires), nous ne pouvons pas non plus accepter l'hypothèse 3 (H3). Finalement, le nombre de relations de mentorat a une influence significative mais négative sur la similitude perçue, sur l'ensemble des fonctions psychologiques ainsi que sur la fonction de guide. Nous ne pouvons donc pas accepter l'hypothèse 4 (H4). 


\section{Tableau 1}

Corrélations ${ }^{\mathrm{a}}$ entre les heures de formation reçues et les réponses du mentoré sur certaines composantes de la relation

\begin{tabular}{|c|c|c|c|c|c|}
\hline \multirow[b]{2}{*}{ Oualité de la relation } & $\begin{array}{c}\text { Heures de } \\
\text { formation } \\
\text { Réseau M } \\
\left(\mathrm{n}=77^{\mathrm{b}}\right)\end{array}$ & \multirow[t]{2}{*}{$\begin{array}{l}\text { Heures de } \\
\text { formation } \\
\text { Autres } \\
(n=51)\end{array}$} & \multirow[t]{2}{*}{$\begin{array}{l}\text { Nb. années } \\
\text { d'expérience } \\
\text { en affaires } \\
(\mathrm{n}=55)\end{array}$} & \multicolumn{2}{|c|}{$\begin{array}{l}\mathrm{Nb} \text { de } \\
\text { relations } \\
\text { mentorat } \\
(\mathrm{n}=66)\end{array}$} \\
\hline & & & & & \\
\hline Confiance envers le mentor & $0,322 * *$ & $0,386 * *$ & 0,128 & 0,059 & \\
\hline Similitude perçue & $0,269 *$ & $0,329 *$ & $-0,063$ & $-0,223$ & $*$ \\
\hline Satisfaction envers le mentor & $-0,001$ & $-0,061$ & 0,036 & 0,162 & \\
\hline \multicolumn{6}{|l|}{ Style du mentor } \\
\hline Approche maïeutique & $0,229 *$ & $0,271 \dagger$ & $-0,016$ & $-0,001$ & \\
\hline Engagement du mentor & $0,242 *$ & 0,167 & $-0,107$ & $-0,149$ & \\
\hline \multicolumn{6}{|l|}{ Fonctions psychologiques } \\
\hline Fonction de réflecteur & $0,216 \dagger$ & 0,206 & $-0,059$ & $-0,226$ & $\dagger$ \\
\hline Fonction de sécurisation & $0,299 *$ & 0,141 & $-0,079$ & $-0,342$ & ** \\
\hline Fonction de motivation & $0,349 * *$ & 0,187 & $-0,046$ & $-0,229$ & $\dagger$ \\
\hline Fonction de confident & $0,236 \dagger$ & 0,156 & 0,053 & $-0,293$ & $*$ \\
\hline \multicolumn{6}{|l|}{ Fonctions reliées à la carrière } \\
\hline Fonction d'intégration & 0,109 & 0,066 & 0,092 & $-0,024$ & \\
\hline Fonction de soutien informat. & 0,055 & $-0,006$ & $-0,111$ & $-0,102$ & \\
\hline Fonction de confrontation & 0,197 & 0,073 & $-0,063$ & $-0,154$ & \\
\hline Fonction de guide & $0,263 *$ & 0,091 & 0,006 & $-0,302$ & $*$ \\
\hline \multicolumn{6}{|l|}{ Fonction de modèle de rôle } \\
\hline Fonction de modèle & $0,238 \dagger$ & 0,069 & 0,087 & $-0,134$ & \\
\hline \multicolumn{6}{|l|}{ Retombée de la relation } \\
\hline Apprentissage du mentoré & $0,209+$ & $-0,004$ & 0,082 & $-0,091$ & \\
\hline
\end{tabular}

${ }^{a}$ Les corrélations de Spearman (non paramétriques) ont été utilisées à cause de la distribution de certaines variables qui ne sont pas normales.

${ }^{\mathrm{b}}$ Certaines données sont manquantes et le plus petit « $\mathrm{n} » \mathrm{a}$ été indiqué

$\dagger=\mathrm{p} \leq 0,10 ; *=\mathrm{p} \leq 0,05, * *=\mathrm{p} \leq 0,01 ; * * *=\mathrm{p} \leq 0,001$;

\subsection{La formation peut-elle annuler l'effet négatif de l'expérience de mentorat?}

Ces résultats ont suscité une grande curiosité et la réflexion des chercheurs. Si la formation a un effet positif mais que l'expérience de mentorat a un effet négatif, est-ce que la formation pourrait annuler l'effet négatif de l'expérience de mentorat ? Cette question de recherche, non prévue initialement, est fondamentale pour les organismes d'accompagnement qui offrent des services de mentorat. Pour y répondre, nous avons créé deux groupes en séparant le nombre de relations de mentorat à la médiane (4 relations) afin de comparer l'effet de la formation chez les mentors ayant peu d'expérience de mentorat (4 relations et moins) avec l'effet de la formation chez les mentors expérimentés ( 5 relations et plus). Les résultats, tel qu'on peut les voir au Tableau 2 , sont éloquents. Comme on peut le constater, dans le groupe des mentors peu expérimentés dans le mentorat, la formation ne permet que d'améliorer la fonction de 
sécurisation du mentoré. Par contre, chez le groupe de mentors expérimentés, la formation permet d'augmenter la confiance et la similitude perçue, de faire adopter un style davantage maïeutique au mentor, de bonifier l'intensité de toutes les fonctions psychologiques ainsi que des fonctions de guide et de modèle, et finalement de bonifier les apprentissages de l'entrepreneur mentoré.

\section{Discussion}

Nous aborderons d'abord la contribution théorique de cette recherche. Par la suite, nous en présenterons les limites, avant de compléter avec les recommandations pour les pouvoirs publics et les organismes de soutien à l'entrepreneuriat qui gèrent des programmes de mentorat.

\subsection{Contribution théorique de la recherche}

Les résultats obtenus indiquent qu'être un bon mentor, cela s'apprend. Les recherches sur le mentorat indiquent que la première retombée du mentorat (retombée proximale) est l'apprentissage issu de la relation (Wanberg et al., 2003).

Sachant l'importance que revêt l'apprentissage pour un entrepreneur novice dans une relation de mentorat, cela démontre l'importance de former les mentors afin de maximiser les retombées chez les entrepreneurs. Au-delà des apprentissages réalisés par le mentoré, les résultats de la formation sont également éloquents, notamment pour mettre en place une relation de qualité avec le mentoré, laquelle lui permettra de générer diverses retombées. Ainsi, il semble que la formation serve de base pour démarrer et entretenir une relation de qualité. Nous avons constaté que les formations dans le Réseau M donnent de meilleurs résultats que les autres qui sont dispensées par d'autres organismes. Ici, tout nous porte à croire que la nature spécifique d'une relation de mentorat pour entrepreneur amène le développement de formations sur mesure (via le Réseau $\mathrm{M}$ ), plutôt que des formations généralistes (via les autres organismes non dédiés à l'entrepreneuriat), lesquelles ne semblent être utiles que pour donner au mentor les moyens de mettre en place une relation de qualité (via la mise en confiance du mentoré et le développement de la similitude perçue) basée sur les questionnements (approche maïeutique).

Nos résultats ne permettent pas d'affirmer que le mentor ayant eu une carrière d'entrepreneur offrira un accompagnement de mentorat de meilleure qualité que ceux qui ont une carrière passée dans la fonction publique ou dans de grandes organisations. Contrairement à nos attentes initiales, il semblerait que la carrière du mentor n'influence pas la relation de mentorat. Ici, il faut préciser quelques limites. Il est possible, pour certains entrepreneurs novices faisant la demande d'être accompagnés, que le passé d'entrepreneur du mentor soit quelque chose d'important à leurs yeux. Du coup, en spécifiant cet aspect au coordonnateur du programme, le jumelage ne s'en ressentira donc pas puisqu'il sera jumelé avec un mentor correspondant au profil recherché. Pour les autres mentorés pour qui la carrière passée du mentor n'a pas d'importance, ils seront donc jumelés avec différents profils, dont des mentors n'ayant pas eu une carrière d'entrepreneur. Ainsi, les résultats semblent indiquer qu'une fois qu'un mentoré accepte le profil de son mentor quant à sa carrière (entrepreneur ou non), cela n'affectera plus d'aucune manière la relation qui s'ensuivra. 
Tableau 2

Corrélations ${ }^{a}$ entre les heures de formation reçues et les réponses du mentoré sur certaines composantes de la relation, selon leur niveau d'expérience de mentorat

\begin{tabular}{|c|c|c|}
\hline & $\begin{array}{r}\text { Heures } \\
\text { Ré }\end{array}$ & $\begin{array}{l}\text { ormation } \\
\mathrm{M}\end{array}$ \\
\hline & $\begin{array}{c}\text { Mentor peu } \\
\text { expérimenté } \\
(\leq 4 \text { relations }) \\
n=23^{b}\end{array}$ & $\begin{array}{c}\text { Mentor } \\
\text { expérimenté } \\
(\geq 5 \text { relations }) \\
n=42\end{array}$ \\
\hline Qualité de la relation & & \\
\hline Confiance envers le mentor & 0,054 & $0,467 * * *$ \\
\hline Similitude perçue & 0,051 & $0,421 * *$ \\
\hline Satisfaction envers le mentor & $-0,018$ & 0,009 \\
\hline Style du mentor & & \\
\hline Approche maïeutique & 0,148 & $0,296 *$ \\
\hline Engagement du mentor & 0,282 & 0,234 \\
\hline Fonctions psychologiques & & \\
\hline Fonction de réflecteur & 0,093 & $0,346 *$ \\
\hline Fonction de sécurisation & $0,407+$ & $0,381 *$ \\
\hline Fonction de motivation & 0,258 & $0,429 * *$ \\
\hline Fonction de confident & 0,171 & $0,345 *$ \\
\hline Fonctions reliées à la carrière & & \\
\hline Fonction d'intégration & 0,074 & 0,112 \\
\hline Fonction de soutien informationnel & $-0,037$ & 0,141 \\
\hline Fonction de confrontation & 0,162 & 0,251 \\
\hline Fonction de guide & 0,299 & $0,364 *$ \\
\hline Fonction de modèle de rôle & & \\
\hline Fonction de modèle & 0,046 & $0,404 * *$ \\
\hline Retombée de la relation & & \\
\hline Apprentissage du mentoré & 0,079 & $0,311 *$ \\
\hline $\begin{array}{l}\text { a Les corrélations de Spearman (non paramétriques) ont été ut } \\
\text { variables qui ne sont pas normales. } \\
\text { va Certaines données sont manquantes et le plus petit «n } » \text { a éte } \\
\dagger=\mathrm{p} \leq 0,10 ; *=\mathrm{p} \leq 0,05, * *=\mathrm{p} \leq 0,01 ; * * *=\mathrm{p} \leq 0,001 ;\end{array}$ & $\begin{array}{l}\text { sées à cause de } 1 \\
\text { ndiqué }\end{array}$ & ition de certaines \\
\hline $\begin{array}{l}\text { L'effet négatif de l'expérience de mentorat est un } \\
\text { littérature traitant de mentorat dans d'autres conter } \\
\text { l'expérience passée en mentorat, nous constatons un } \\
\text { des fonctions psychologiques du mentor et de la } \\
\text { similitude perçue. Ces résultats laissent penser que d } \\
\text { être moins confiants dans leur capacité à accompa } \\
\text { novices. Du coup, ils seraient davantage attentifs à } \\
\text { concernent les dimensions psychologiques de l'acce } \\
\text { pourraient être moins attentifs aux besoins psyc } \\
\text { concentrer davantage sur des questions relatives à l' }\end{array}$ & $\begin{array}{l}\text { ésultat importa } \\
\text { es a rapporté d } \\
\text { ffet négatif, en } \\
\text { nction de guid } \\
\text { ns les débuts, le } \\
\text { ner correcteme } \\
\text { leurs besoins, } \\
\text { npagnement. A }\end{array}$ & $\begin{array}{l}\text { souligner. Si la } \\
\text { effets positifs de } \\
\text { iculier au niveau } \\
\text { insi que dans la } \\
\text { entors pourraient } \\
\text { es entrepreneurs } \\
\text { mment ceux qui } \\
\text { l'expérience, ils } \\
\text { epreneurs et se }\end{array}$ \\
\hline
\end{tabular}


Quant à l'interaction entre la formation et l'expérience, les résultats obtenus permettent de mieux comprendre les mécanismes pour optimiser les retombées pour les entrepreneurs novices. Ainsi, la formation pour les mentors n'est que marginalement utile pour les nouveaux mentors alors qu'elle est fondamentale pour les mentors expérimentés, permettant de neutraliser l'effet négatif de l'expérience accumulé. Ainsi, il semblerait que la formation ferait en sorte de remettre en question les méthodes d'intervention des mentors et de les garder «alertes » à l'égard des aspects psychosociaux d'un accompagnement de type mentorat. Sans formation continue, la qualité de l'intervention des mentors pourrait diminuer sur ces aspects.

\subsection{Limites à l'étude}

Les résultats obtenus possèdent une limite importante : les formations les plus utiles n'ont pas été identifiées. Ainsi, nous savons que la formation a un effet majeur mais il est impossible de cibler des formations adéquates ou certaines qui seraient plus pertinentes que les autres. On peut facilement penser à certaines formations de base à propos du rôle du mentor, incluant les styles les plus efficaces, ainsi que d'autres pour permettre aux mentors de développer l'art de poser des questions au mentoré pour le faire cheminer dans ses réflexions et lui permettre de développer des schémas cognitifs d'experts, par exemple (pour plus de détails sur ce concept, voir Baron et Ensley, 2006). Aussi, rappelons que toutes les analyses en dyade ne concernent jamais plus de 78 cas au total. Cela pourrait causer une incapacité de voir une relation significative (dans les données) là où elle devrait être observée (dans la réalité), ce qui correspond à une erreur de type II, causé par la faiblesse de la puissance des tests effectués. C'est d'ailleurs pour cette raison que les seuils de signification à $\mathrm{p} \leq 0,10$ ont été indiqués. Malgré cette limite, les résultats obtenus par les analyses en dyade sont d'autant plus valides puisque la relation est significative malgré le petit nombre de cas. Une autre limite concerne la notion de temps, qui n'est pas considérée. En effet, une relation de mentorat évolue dans le temps. L'enquête auprès des mentorés en 2008 a recueilli tous les mentorés intéressés à répondre au questionnaire, certains étant au début de leur relation et pour d'autres, elle était terminée. En jumelant en dyade avec les mentors de 2010, cela pourrait induire certaines distorsions quant à la relation de cause à effet entre la formation des mentors et la relation de mentorat étudiée. Cela amène à suggérer une recherche longitudinale, en dyade, pour ainsi constater l'effet du mentorat dans le temps et en fonction des caractéristiques du mentor, notamment de la formation reçue.

\subsection{Recommandations aux pouvoirs publics et aux organismes de soutien aux entrepreneurs}

Les résultats obtenus sont d'une importance capitale pour les organismes d'accompagnement qui offrent un service de mentorat aux entrepreneurs novices. D'abord, dans la perspective où le mentoré accepte le profil de carrière du mentor, il n'est pas nécessaire que celui-ci ait été entrepreneur par le passé, une expérience connexe du monde des affaires peut très bien faire l'affaire. Cette connaissance permet de clarifier certains mythes véhiculés dans le milieu pratique, dont celui que seul un mentor ayant été lui-même en affaires devrait accompagner un autre entrepreneur. Cela ne semble donc pas être le cas. 
Au niveau du type de formation à dispenser aux mentors, bien que nos analyses n'aient pu apporter un éclairage à ce niveau, nous constatons que les formations dédiées et spécifiques aux entrepreneurs seraient plus adaptées pour améliorer un plus vaste éventail de dimensions relatives à une relation de mentorat (qualité de la relation, style, fonctions et retombées). Ainsi, des formations de base en communication et sur la manière d'établir une relation de qualité et d'écoute, basée sur les questionnements, peuvent être dispensées par différents organismes, même si ceux-ci n'ont pas une connaissance fine des réalités des entrepreneurs novices. Par contre, pour aller plus loin, notamment en ce qui concerne les aspects psychosociaux liés à l'entrepreneuriat (et leurs corolaires, les fonctions psychologiques du mentor), une telle connaissance est nécessaire et les formateurs doivent être compétents à cet égard lorsqu'ils développeront des formations aux mentors.

Si les résultats obtenus indiquent l'effet positif de la formation, ce résultat ne doit pas forcément indiquer que seule la formation de type «transmission du savoir » devrait être dispensée pour neutraliser l'effet négatif de l'expérience des mentors. À cet effet, une démarche telle la formation d'un groupe de codéveloppement professionnel (Payette et Champagne, 1997) pour les mentors pourrait être appropriée. À cet égard, les mentors du Réseau M de la Fondation de l'entrepreneurship sont regroupés dans des cellules régionales où ils peuvent échanger sur leur pratique, grâce à la coordination d'un chefmentor, élu par ses pairs. En étant aux faits des résultats de cette étude, de tels groupes pourraient remplacer avantageusement la formation traditionnelle, de type transmission des savoirs, bien que nos résultats ne permettent pas de le vérifier.

Si la formation des mentors s'avère importante, celle-ci est davantage utile auprès des mentors ayant une certaine expérience en accompagnement. Bien que cela n'indique pas forcément qu'il ne faille pas former des mentors qui pourraient avoir certaines carences initiales, nous constatons que, globalement, la formation permet d'annuler l'effet négatif de l'expérience accumulée des mentors. Cela indique clairement aux organismes qui dispensent ces services de la nécessité de mettre en place de la formation continue pour les mentors. À défaut d'investissement à cet égard, la qualité des interventions pourrait diminuer, en particulier dans le soutien psychosocial offert aux novices par les mentors.

\section{Conclusion}

Notre étude, qui porte sur le lien entre la formation des mentors et les résultats pour le mentorés en termes d'efficacité de la relation et d'apprentissage, a permis d'identifier l'un des facteurs de succès du processus de mentorat entrepreneurial, soit la formation donnée aux mentors. Nous savons qu'il n'est pas le seul, mais il nous semble important pour les dispositifs de soutien à l'entrepreneuriat ayant recours au mentorat puisqu'ils peuvent intervenir à cet égard sans entrer dans l'intimité de la relation et en laissant un degré de liberté au mentor et au mentoré dans la gestion de celle-ci. Nos résultats montrent l'importance de la formation des mentors surtout concernant le développement de leurs compétences relationnelles et, en particulier pour les mentors accumulant de l'expérience d'accompagnement. Les formations leur permettent de mieux utiliser ou transmettre leur expérience, et d'éviter les comportements nuisibles. Elle donne également un cadre à la fois éthique et relationnel informant les mentors sur leurs rôles, responsabilités, les limites 
de celles-ci et les règles du jeu. Elle permet aussi aux mentors de développer des compétences d'écoute, d'empathie, et de pratique maïeutique. La formation n'est qu'un des facteurs clés du processus de mentorat entrepreneurial, nous suggérons donc l'approfondissement des recherches sur le processus global et les autres éléments clés de celui-ci, notamment ceux gérés par les coordonnateurs de programmes d'accompagnement. 


\section{Références}

ALLEN, T. D. et EBY, L. T. (2003), « Relationship effectiveness for mentors: Factors associated with learning and quality », Journal of Management, vol. 29, n 4, p. 469-486. ALLEN, T. D. et EBY, L. T. (2004), « Factors Related to Mentor Reports of Mentoring Functions Provided: Gender and Relational Characteristics », Sex Roles, vol. 50, n 1/2, p. 129-139.

ALLEN, T. D., FINKELSTEIN, L. M. et POTEET, M. L. (2009), Designing workplace mentoring programs: an evidence-based approach, Chichester, U.K., Wiley-Blackwell, $200 \mathrm{p}$.

ARMSTRONG, J. S. et OVERTON, T. S. (1977), « Estimating nonresponse bias in mail surveys ", Journal of Marketing Research, vol. 14, n 3, p. 396-402.

BARON, R. A. et ENSLEY, M. D. (2006), « Opportunity Recognition as the Detection of Meaningful Patterns: Evidence from Comparisons of Novice and Experienced Entrepreneurs », Management Science, vol. 52, no 9, p. 1331-1344.

BAUER, T. N. (1999), « Perceived mentoring fairness: Relationships with gender, mentoring type, mentoring experience, and mentoring needs », Sex roles, vol. 40, n 3, p. 211-225.

BAUGH, S. G. et FAGENSON-ELAND, E. (2007), « Formal mentoring programs: A "poor cousin" to informal relationships », dans B. R. Ragins et K. E. Kram (dir.), The handbook of mentoring at work: Theory, research, and practice, Los Angeles, USA, Sage Publications, p. 249-271.

CHOUEKE, R. et ARMSTRONG, R. (1998), « The learning organisation in small and medium-sized enterprises: A destination or a journey? ", International Journal of Entrepreneurial Behaviour \& Research, vol. 4, n 2, p. 129-140.

CLUTTERBUCK, D. et MEGGINSON, D. (1999), Mentoring Executives and Directors, Oxford, UK, Butterworth-Heinemann, 167 p.

COPE, J. et WATTS, G. (2000), « Learning by doing - An exploration of experience, critical incidents and reflection in entrepreneurial learning », International Journal of Entrepreneurial Behaviour \& Research, vol. 6, n 3, p. 104-124.

COUTERET, P., ST-JEAN, E. et AUDET, J. (2006), « Le mentorat : conditions de réussite de ce mode d'accompagnement de l'entrepreneur ». 23 ème conférence $d u$ $C C P M E / C C S B E$, Trois-Rivières, Québec, 28-30 septembre.

CRANWELl-WARD, J., BOSSONS, P. et GOVER, S. (2004), Mentoring: A Henley review of best practice, New-York, USA, Palgrave Macmillan, $256 \mathrm{p}$.

CULL, J. (2006), " Mentoring young entrepreneurs: What leads to success? ", International Journal of Evidence Based Coaching and Mentoring, vol. 4, n ${ }^{\mathrm{o}}$ 2, p. 8-18.

DEAKINS, D., GRAHAM, L., SULLIVAN, R. et WHITTAM, G. (1998), « New venture support: an analysis of mentoring support for new and early stage entrepreneurs ", Journal of Small Business and Enterprise Development, vol. 5, $\mathrm{n}^{\mathrm{o}}$ 2, p. 151-161.

DESCHAMPS, B., FATIEN, P. et GEINDRE, S. (2010), " Accompagner le repreneur d'entreprise : conduire, escorter mais aussi guider », Gestion 2000, vol. 27, nº 3, p. 7788 .

EBY, L. T. (2007), « Understanding relational problems in mentoring », dans B. R. Ragins et K. E. Kram (dir.), The handbook of mentoring at work: Theory, research, and practice, Los Angeles, USA. Vol. Sage Publications, p. 323-344. 
EBY, L. T., BUTTS, M., LOCKWOOD, A. et SHANA, S. A. (2004), « Protégés' negative mentoring experiences: Construct development and nomological validation », Personnel Psychology, vol. 57, $\mathrm{n}^{\circ}$ 2, p. 411-447.

ENSHER, E. A., GRANT-VALLONE, E. J. et MARELICH, W. D. (2002), « Effects of Perceived Attitudinal and Demographic Similarity on Protégés' Support and Satisfaction Gained From Their Mentoring Relationships », Journal of Applied Social Psychology, vol. 32, no 7, p. 1407-1430.

ENSHER, E. A. et MURPHY, S. E. (1997), « Effects of Race, Gender, Perceived Similarity, and Contact on Mentor Relationships », Journal of Vocational Behavior, vol. $50, \mathrm{n}^{\circ} 3$, p. $460-481$.

FAGENSON-ELAND, E. A., MARKS, M. A. et AMENDOLA, K. L. (1997), « Perceptions of Mentoring Relationships "), Journal of Vocational Behavior, vol. 51, $\mathrm{n}^{\circ} 1$, p. 29-42.

FLORÉN, H. (2003), «Collaborative approaches to management learning in small firms », Journal of Workplace Learning, vol. 15, no 5, p. 203-216.

GRAVELLS, J. (2006), " Mentoring start-up entrepreneurs in the East Midlands Troubleshooters and trusted friends ", The International Journal of Mentoring and Coaching, vol. 4, n ${ }^{\mathrm{o}}$ 2, p. 3-23.

HAGGARD, D. L., DOUGHERTY, T. W., TURBAN, D. B. et WILBANKS, J. E. (2011), «Who Is a Mentor? A Review of Evolving Definitions and Implications for Research », Journal of Management, vol. 37, $\mathrm{n}^{\circ}$ 1, p. 280-304.

HATTINGH, M., COETZEE, M. et SCHREUDER, D. (2007), « Implementing and sustaining mentoring programmes: A review of the application of best practices in the South African organisational context ", SA Journal of Human Resource Management, vol. 3, no 3, p. 40-48.

KRAM, K. E. (1985), Mentoring at work: Developmental relationships in organizational life Glenview, IL, Scott Foresman, 252 p.

LANKAU, M. J., RIORDAN, C. M. et THOMAS, C. H. (2005), " The effects of similarity and liking in formal relationships between mentors and protégés », Journal of Vocational Behavior, vol. 67, $\mathrm{n}^{\circ}$ 2, p. 252-265.

NANDRAM, S. S. (2003), "Entrepreneurs' need for mentoring and their individual differences ». ICSB 48 ${ }^{\text {th }}$ World Conference, Belfast, Ireland, June, 15-18.

OZGEN, E. et BARON, R. A. (2007), « Social sources of information in opportunity recognition: Effects of mentors, industry networks, and professional forums », Journal of Business Venturing, vol. 22, nº 2, p. 174-192.

PAUL, M. (2004), L'accompagnement : une posture professionnelle spécifique, Paris, France, L'Harmattan, $356 \mathrm{p}$.

PAYETTE, A. et CHAMPAGNE, C. (1997), Le groupe de codéveloppement professionnel, Ste-Foy, Québec, Presses de l'Université du Québec.

PELLEGRINI, E. K. et SCANDURA, T. A. (2005), "Construct equivalence across groups: an unexplored issue in mentoring research ", Educational and Psychological Measurement, vol. $65, \mathrm{n}^{\mathrm{o}}$ 2, p. 323-335.

RAGINS, B. R. (1997), « Antecedents of diversified mentoring relationships », Journal of Vocational Behavior, vol. 51, no 1, p. 90-109.

RAGINS, B. R. et COTTON, J. L. (1999), " Mentor functions and outcomes: A comparison of men and women in formal and informal mentoring relationships », Journal of Applied Psychology, vol. 84, nº 4, p. 529-550. 
RAGINS, B. R., COTTON, J. L. et MILLER, J. S. (2000), « Marginal mentoring: The effects of type of mentor, quality of relationship, and program design on work and career attitudes », Academy of Management Journal, vol. 43, nº 6, p. 1177-1184.

REMPEL, J. K. et HOLMES, J. G. (1986), « How do I trust thee ? », Psychology Today, vol. $20, n^{\circ} 2$, p. $28-34$.

SCANDURA, T. A. et WILLIAMS, E. A. (2001), « An investigation of the moderating effects of gender on the relationships between mentorship initiation and protégé perceptions of mentoring functions », Journal of Vocational Behavior, vol. 59, nº 3, p. 342-363.

SIMON, S. A. et EBY, L. T. (2003), « A typology of negative mentoring experiences: A multidimensional scaling study », Human Relations, vol. 56, nº 9, p. 1083-1109.

ST-JEAN, E. (2010), « Les fonctions du mentor de l'entrepreneur novice », Revue de l'entrepreneuriat, vol. 9, $\mathrm{n}^{\circ}$ 2, p. 28-49.

ST-JEAN, E. et AUDET, J. (2011), « The Effect of Mentor Intervention Style in Novice Entrepreneur Mentoring Relationships ». Academy of Management Annual Meeting, San Antonio (USA), August $12^{\text {th }}-16^{\text {th }}$.

ST-JEAN, E. et AUDET, J. (2012), « The role of mentoring in the learning development of the novice entrepreneur », International Entrepreneurship and Management Journal, vol. 8, $\mathrm{n}^{\mathrm{o}} 1$, p. 119-140.

UCBASARAN, D., WESTHEAD, P. et WRIGHT, M. (2009), " The extent and nature of opportunity identification by experienced entrepreneurs », Journal of Business Venturing, vol. 24, no 2, p. 99-115.

WANBERG, C. R., KAMMEYER-MUELLER, J. et MARCHESE, M. (2006), « Mentor and protégé predictor and outcomes of mentoring in a formal mentoring program », Journal of Vocational Behavior, vol. 69, n 3, p. 410-423.

WANBERG, C. R., WELSH, E. T. et HEZLETT, S. A. (2003), « Mentoring research: A review and dynamic process model », dans J. J. Martocchio et G. R. Ferris (dir.), Research in personnel and human resources management, Oxford, UK, Elsevier Science Ltd. Vol. 22, p. 39-124.

WIKHOLM, J., HENNINGSON, T. et HULTMAN, C. M. (2005), " Demand of mentoring among new starters ». ICSB $50^{\text {th }}$ World Conference, Washington, DC.

WILSON, J. A. et ELMAN, N. S. (1990), " Organizational benefits of mentoring », Academy of Management Executive, vol. 4, nº 4, p. 88-94. 


\section{Annexe 1}

Tableau 3 - Détail des mesures utilisées dans le questionnaire du mentoré

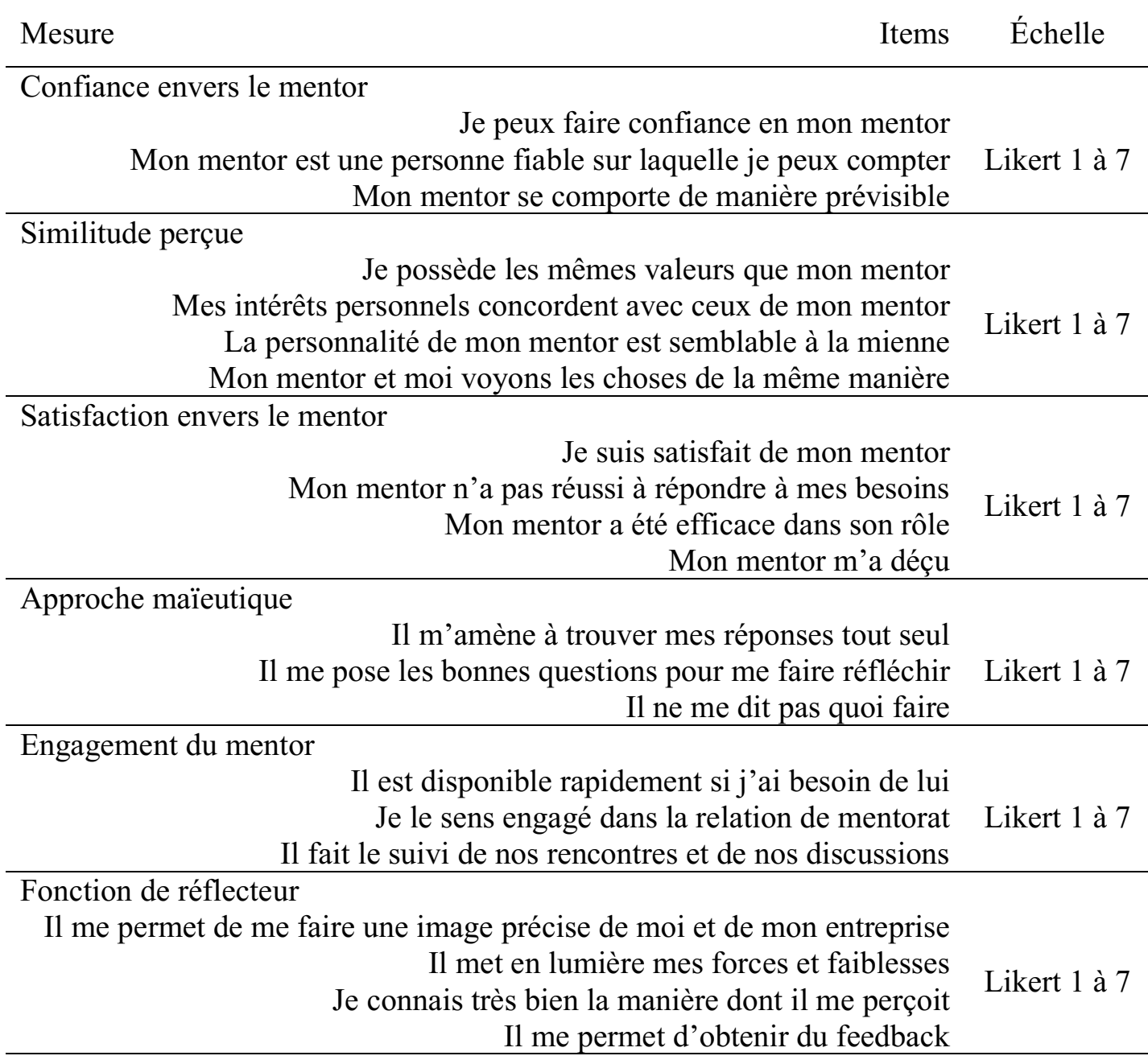

Fonction de sécurisation

Il me calme lorsque je suis tendu Il me sécurise Likert 1 à 7

Fonction de motivation

Il m'aide à relativiser mes problèmes

Je sens qu'il a confiance en mes capacités

Il me motive

Il m'encourage à persévérer

Likert 1 à 7

Il croit que je peux réussir comme entrepreneur

Fonction de confident

Il est un bon confident

Il est une personne à qui je peux me confier

Je le considère comme un ami

Likert 1 à 7

Il est plus qu'une simple connaissance d'affaires

Fonction d'intégration

Il me met en relation avec des gens qu'il connaît

Il me présente à des personnes de son entourage

Likert 1 à 7 
Il me suggère des individus qui peuvent m'aider Il est prêt à me faire bénéficier de ses contacts

Fonction de soutien informationnel

Il me fournit des informations et des renseignements reliés au monde des affaires

Il partage ses savoirs et ses connaissances Likert 1 à 7

Il me donne des informations techniques Il me fait profiter de l'expertise qu'il possède

Fonction de confrontation

Il met en lumière les conséquences liées à mes décisions Il n'hésiterait pas à me contredire s'il n'était pas d'accord Il m'oblige à démontrer la justesse de mes idées Likert 1 à 7 Il critique mes décisions de manière constructive

Fonction de guide

Il me suggère de nouvelles options

Il me propose d'autres points de vue

Il me donne des conseils à propos de mes problèmes

Likert 1 à 7

Il m'aide à clarifier le problème vécu

Fonction de modèle

Il me sert de modèle

Il m'expose ses réussites et ses échecs

Il est un bon exemple d'entrepreneur

Likert 1 à 7

Il me fait part de ses expériences d'affaires et de vie

Apprentissage du mentoré

J'ai appris beaucoup de mon mentor

Mon mentor m'a apporté de nouvelles perspectives sur plusieurs choses

Mon mentor et moi avons appris ensemble, en collaboration

Il y a un apprentissage réciproque qui s'est produit avec mon mentor

Likert 1 à 7

Mon mentor a partagé avec moi beaucoup d'informations qui m'ont aidé

dans mon développement professionnel 